\title{
Suppression of tumor cell proliferation by quinine via the inhibition of the tumor necrosis factor receptor-associated factor 6 -AKT interaction
}

\author{
WENJUAN LIU, YONGHAO QI, LINGYU LIU, YU TANG, JING WEI and LIJUN ZHOU \\ Department of Molecular and Cellular Pharmacology, School of Pharmaceutical Science and Technology, \\ Tianjin University, Tianjin 300072, P.R. China
}

Received May 8, 2015; Accepted April 6, 2016

DOI: $10.3892 / \mathrm{mmr} .2016 .5492$

\begin{abstract}
Protein kinase B (AKT), is a pivotal component of pathways associated with cell survival, metabolism, invasion and metastasis. AKT mediates anti-apoptotic and proliferative signaling in response to essential cytokines. Tumor necrosis factor receptor-associated factor (TRAF)6, an E3 ubiquitin ligase, has been shown to ubiquitylate, as well as activate AKT. The present study used computational methods to determine the relevant amino acid residues at the binding site of TRAF6 and selected small molecules, which may bind to TRAF6. An ex vivo assay was performed to determine their antitumor activities and the possible mechanism of action. Quinine, a natural alkaloid that is well-known for its therapeutic treatment of malaria, exhibited a distinct antiproliferative and pro-apoptotic effect in HeLa and A549 tumor cell lines via the inhibition of the antiapoptotic protein, B-cell lymphoma (BCL)-2, and activation of the pro-apoptotic factor, BCL-2-associated X protein. Quinine inhibited the lipopolysaccharide (LPS)-induced activation of AKT by inhibiting its phosphorylation at Thr-308 and Ser-473, and reversing LPS-induced proliferation. These results suggested that the
\end{abstract}

Correspondence to: Miss. Lijun Zhou or Miss. Jing Wei, Department of Molecular and Cellular Pharmacology, School of Pharmaceutical Science and Technology, Tianjin University, 92 Weijin Road, Nankai, Tianjin 300072, P.R. China

E-mail: lijunzhou@tju.edu.cn

E-mail: betty_wj@tju.edu.cn

Abbreviations: BCL, B-cell lymphoma; BAD, BCL-2-associated death domain; BAX, BCL-2-associated $\mathrm{X}$ protein; Co-IP, co-immunoprecipitation; FBS, fetal bovine serum; FITC, fluorescein isothiocyanate; PI, propidium iodide; HRP, horseradish peroxidase;

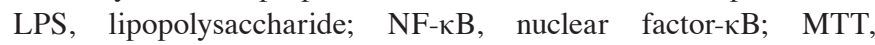
3-(4,5-dimethylthiazol-2-yl)-2,5-diphenyltetrazolium bromide; PDK, phosphoinositide-dependentkinase; RIPA,radioimmunoprecipitation assay; TRAF6, tumor necrosis factor receptor-associated factor 6 .

Key words: quinine, tumor necrosis factor receptor-associated factor 6, protein kinase B, cancer, proliferation, apoptosis inhibition of AKT activation via targeting of TRAF6 with quinine may be a viable anticancer therapeutic approach and a successful example of the alternative use of the original therapeutic properties of this well-known natural product.

\section{Introduction}

Phosphatidylinositol-4,5-bisphosphate 3-kinase (PI3K) and protein kinase B (AKT) are involved in major pro-survival signaling pathways in cancer cells. While excessive proliferation and survival of aberrant cells is suppressed in normal homeostasis, in tumor cells, this is overridden by the upregulation of antiapoptotic proteins and other cell death-associated factors. In particular, AKT regulates cell survival, metabolism, invasion and metastasis, and is also pivotal role in antiapoptotic and antiproliferative signaling in response to essential cytokines. AKT-dependent cell survival is mediated through the induction of antiapoptotic gene expression (1), including members of the forkhead and B-cell lymphoma (BCL)-2 family, as well as the nuclear factor (NF)- $\mathrm{\kappa B}$. It is known that the BCL-2 family of proteins regulates apoptosis through modulating mitochondrial permeability (2). In addition, AKT-dependent degradation of the inhibitor of NF- $\kappa B$ leads to $\mathrm{NF}-\mathrm{\kappa B}$ translocation from the cytoplasm to the nucleus where it promotes cell survival by regulating the expression levels of target genes. AKT can also inhibit p53, which is a tumor suppressor protein that induces apoptosis, senescence and cell cycle arrest (3).

Consequently, drugs designed to target AKT have become an important and viable approach in the development of cancer therapeutics with a number of AKT inhibitors already being used in clinical trials. Additionally, it has become increasingly apparent that inhibiting AKT via the inhibition of upstream signals can be equally as significant as direct targeting of this kinase. More specifically, tumor necrosis factor receptor-associated factor (TRAF)6, which is an E3 ubiquitin ligase, has been shown to ubiquitylate and activate AKT (4), and it also serves an essential role in regulating the NF- $\kappa \mathrm{B}$ and mitogen-activated protein kinase signaling cascades. TRAF6 consists of a conserved C-terminal domain that regulates interactions with upstream signaling proteins, and an $\mathrm{N}$-terminal domain that comprises the core of the ubiquitin ligase catalytic domain (5). 
In the presence of E2 ubiquitin-conjugating enzymes, TRAF6 catalyzes the transfer of polyubiquitin chains to the substrates (6). Notably, TRAF6 mediates K63-directed ubiquitylation of AKT, and this type of modification is associated with functional changes in the target substrate in contrast to classical K48-directed ubiquitylation, which leads to substrate degradation.

Additionally, in the context of insulin-like growth factor-1 stimulation, overexpression of TRAF6 enhances AKT phosphorylation and activation. By contrast, AKT phosphorylation at Thr-308 and Ser-473 was inhibited in TRAF6-deficient mouse embryonic fibroblasts (4). TRAF6 is recruited by the phosphorylated discoidina neuropilin-like membrane protein discoidin, CUB and LCCL domain-containing protein 2 , leading to increased E3 ubiquitin ligase activity and subsequent activation of AKT, and accelerating epidermal growth factor receptor-driven tumorigenesis (7). As a result of the close association between TRAF6 and AKT, the present study speculated that AKT can be indirectly targeted by developing small molecules that can bind to TRAF6 and disrupt its interaction with AKT, and that this may be a promising approach in cancer therapeutics. To determine this, the present study performed a computational docking study to screen small molecules, which can bind to TRAF6 in regions critical for its interaction with AKT (residues 50-159, RING and zinc finger domains). The present study found that quinine, a member of well-known Cinchona alkaloids, has a high affinity for TRAF6 and may indeed induce apoptosis, as well as reduce cell viability. The underlying mechanism by which quinine inhibited AKT activation was also examined using a range of ex vivo cellular assays.

\section{Materials and methods}

Materials. A549 human lung adenocarcinoma and HeLa cervical cancer cell lines were kindly provided by Dr. Yaozhou Zhang (Tianjin International Joint Academy of Biomedicine, Tianjin, China). RPMI-1640 medium and fetal bovine serum (FBS) were obtained from Tianjin HOPE Biotechnology Co., Ltd. (Tianjin, China). Rabbit polyclonal antibodies against BCL-2 (cat. no. 2876), BCL-2-associated X protein (BAX; cat. no. 2772), total AKT (cat. no. 4691), phosphorylated (p-)AKT (Thr308; cat. no. 4056s) and p-AKT (Ser473; cat.no.4051s) were purchased from Cell Signaling Technology, Inc. (Danvers, MA, USA). Rabbit polyclonal antibody against $\beta$-actin (cat. no. PR0255) was purchased from Beijing Zhongshan Golden Bridge Biotechnology Co., Ltd. (Beijing, China). Horseradish peroxidase (HRP)-conjugated goat polyclonal antibody against rabbit and mouse (cat. no. M21003) was obtained from Shanghai Abmart Biotechnology Co., Ltd. (Shanghai, China). Radioimmunoprecipitation assay (RIPA) buffer and protease inhibitor cocktail were purchased from Beijing Kangwei Biotechnology Co., Ltd. (Beijing, China). Phosphatase inhibitor cocktail and lipopolysaccharide (LPS) were purchased from Sigma-Aldrich (St. Louis, MO, USA). Quinine was purchased from Tianjin Xiensi Biochemical Technology Co., Ltd. (Tianjin, China). Cis-platinum was purchased from Jiangsu Hansoh Pharmaceutical Co., Ltd. (Tianjin, China). Protein A sepharose beads were purchased from Beijing Kangwei Biotechnology Co., Ltd. (Pierce,
Rockford, IL, USA). All chemicals and solvents were of analytical grade.

Virtual screening. A three-dimensional (3D) structure library containing 1,792 compounds was established for the virtual screen. Structures of these compounds were generated using a two-dimensional (2D)/3D editor-sketcher and were minimized to a local energy minimum using the CHARMm-like force field implemented within with Catalyst 4.11 software (8). The 3D structure of TRAF6 (residues 50-159, RING and zinc finger domains) was retrieved from the Protein Data Bank as the docking receptor (http://www.rcsb.org/pdb; accession no. 3HCT). Both the TRAF6 crystal structure and candidate ligands were prepared using AutoDock Tools v.1.5.2 software (http://autodock.scripps.edu).

For virtual screening, flexible ligands and rigid receptor docking calculations were performed with the AutoDock4.10 software package using the following parameters: The Lamarckian genetic algorithm; a population size of 300; energy evaluations of 25,000,000; search runs of 100 . The docking area was defined as a dimension of 60x60x60 points with grid spacing of $0.375 \AA$. The grid box was centered on the binding site of the ubiquitin-conjugating E2 enzyme, Ubc13. The results were ranked, according to the binding free energy. The conformations from the docking experiments were analyzed using Chimera software (9), which also identified the hydrophobic interactions between the receptor and the ligands.

Cell culture. A549 and HeLa cells were cultured in RPMI-1640 medium, supplemented with $10 \% \mathrm{FBS}$ at $37^{\circ} \mathrm{C}$ in a humidified atmosphere of $5 \% \mathrm{CO}_{2}$.

Cell viability assay. The effect of quinine on HeLa and A549 cell viability and proliferation was determined using a 3-(4,5-dimethylthiazol-2-yl)-2,5-diphenyltetrazolium bromide (MTT) assay. Briefly, $200 \mu \mathrm{l} \mathrm{HeLa} \mathrm{or} \mathrm{A549} \mathrm{cell}$ suspensions were seeded into 96 -well plates at densities of 4 and $3 \times 10^{3}$ cells/well, respectively. The cells were allowed to adhere overnight and were subsequently treated with LPS $(20 \mu \mathrm{g} / \mathrm{ml})$ in the absence or presence of quinine at various concentrations $(1,5,10,25,50,100,250$ and $500 \mu \mathrm{M})$. Cis-platinum $(16.7 \mu \mathrm{M})$ was used as a positive control. Cell viability was assessed by adding $20 \mu \mathrm{l}$ of $5 \mathrm{mg} / \mathrm{ml}$ MTT to each well and incubating for 4-6 h. The medium was replaced with $150 \mu$ l dimethyl sulfoxide, which was stirred to dissolve the formazan crystals. The absorbance (A) was measured at $490 \mathrm{~nm}$ using an ST-360 microplate reader (Shanghai Kehua Bio-engineering Co., Ltd., Shanghai, China). The survival rate of each group of cells was calculated relative to the vehicle-treated control cells (designated as 100\%). Cell inhibition ratio was calculated using the following formula: Cell inhibition ration $=\left(\mathrm{A}_{\text {control }}-\mathrm{A}_{\text {treated }} / \mathrm{A}_{\text {control })} \mathrm{x} 100\right.$.

Detection of apoptosis by flow cytometry. Apoptosis was assessed by flow cytometry using an Apoptosis Detection kit (Becton-Dickinson, Franklin Lakes, NJ, USA), according to the manufacturer's protocol. Briefly, HeLa cells were seeded into a $6 \mathrm{~cm}$ plate at a density of $6 \times 10^{5}$ cells/well and allowed to attach overnight. The medium was replaced with 
A

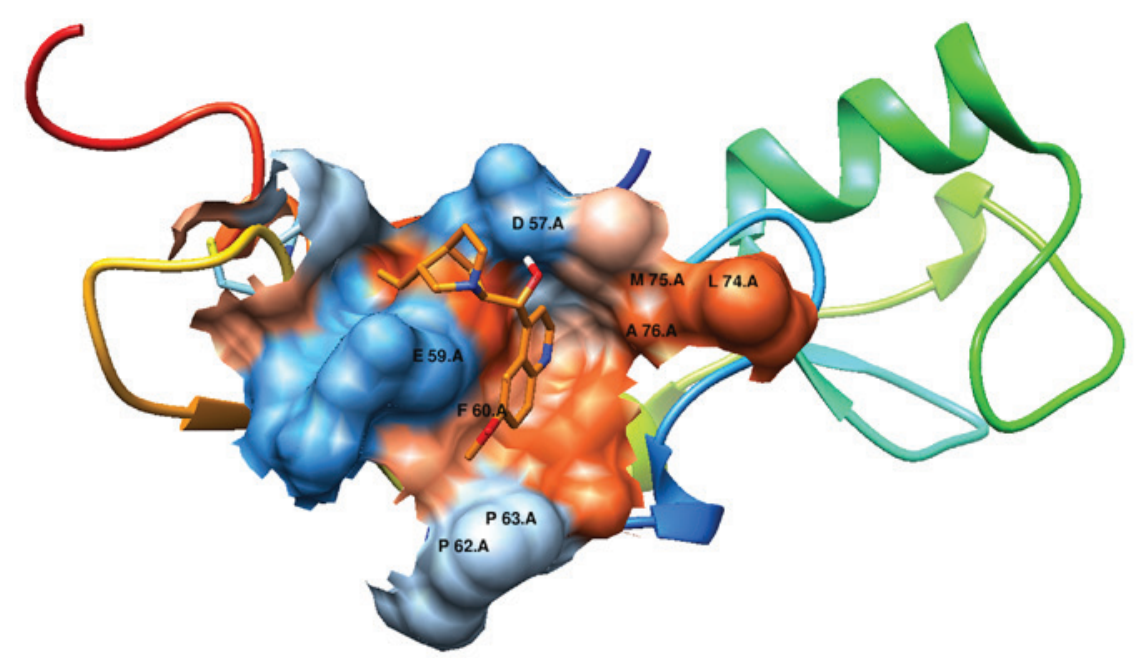

B<smiles>C=CC1CCN([C@@H](O)c2ccnc3ccc(OC)cc23)C1</smiles><smiles>C=CC1CN2CCC1CC2[C@H](O)c1ccnc2ccc(OC)cc12</smiles>

\section{quinidine}<smiles>C=C[C@@H]1CCN([C@H](O)c2ccnc3ccccc23)C1</smiles>

cinchonidine<smiles>C=CC1CN2CCC1CN2C(O)c1ccnc2ccccc12</smiles>

\section{cinchonine}

Figure 1. (A) Predicted binding model of quinine to tumor necrosis factor receptor-associated factor 6 and (B) the chemical structure of quinine and other known cinchona alkaloids.

fresh medium containing various concentrations of quinine $(100,150$ or $200 \mu \mathrm{M})$ for $48 \mathrm{~h}$ at $37^{\circ} \mathrm{C}$. The cells were collected and resuspended in $1 \mathrm{X}$ binding buffer at a concentration of $1 \times 10^{6}$ cells $/ \mathrm{ml}$, and $100 \mu \mathrm{l}$ of the cell suspension was transferred to a $5 \mathrm{ml}$ culture tube, to which $5 \mu \mathrm{l}$ annexin $\mathrm{V}$-fluorescein isothiocyanate (FITC) and propidium iodide (PI) were added (from the Apoptosis Detection kit). The mixture was incubated in the dark for $15 \mathrm{~min}$ at room temperature $\left(25^{\circ} \mathrm{C}\right)$. The apoptotic index was immediately determined with a FACSCalibur instrument (BD Biosciences, Franklin Lakes, NJ, USA). Cell Quest Pro software v5.1 (BD Biosciences) was used to analyze flow cytometry data.

Western blot analysis. Western blotting was performed according to a standard protocol. Briefly, HeLa and A549 cells at $60-70 \%$ confluence were incubated with $150 \mu \mathrm{M}$ quinine. The cells were rinsed with ice-cold phosphate-buffered saline and resuspended in RIPA buffer, supplemented with 
phenylmethylsulfonyl fluoride and phosphatase inhibitors. Following an incubation on ice for $30 \mathrm{~min}$, the lysates were centrifuged at $12,000 \mathrm{x}$ g for $10 \mathrm{~min}$ and the supernatant was collected. Protein concentrations were determined using a bicinchoninic acid protein assay kit (CWBIO, Beijing, China) and $40 \mu \mathrm{g}$ protein was resolved by $3 \%$ sodium dodecyl sulfate-polyacrylamide gel electrophoresis. The proteins were transferred onto a polyvinylidene difluoride membrane (EMD Millipore, Bedford, MA, USA), which was blocked with a solution of $5 \%$ non-fat dry milk or $5 \%$ bovine serum albumin in Tris-buffered saline (10 mM Tris- $\mathrm{HCl}$ and $150 \mathrm{mM}$ $\mathrm{NaCl}$ ) with $0.05 \%$ Tween-20. After $2 \mathrm{~h}$, the membranes were washed and incubated with primary antibodies against $\beta$-actin $(1: 1,500)$, AKT $(1: 1,000)$, p-AKT $(1: 1,000)$, BCL-2 $(1: 1,000)$, $\operatorname{BAX}(1: 1,000)$, and TRAF6 $(1: 1,000)$ overnight at $4^{\circ} \mathrm{C}$, followed by washing and incubation with HRP-conjugated goat anti-rabbit or anti-mouse immunoglobulin $\mathrm{G}(1: 10,000)$ for $1 \mathrm{~h}$ at $25^{\circ} \mathrm{C}$. Antibody binding was detected using an enhanced chemiluminescence kit (CWBIO), according to the manufacturer's protocol. Image J software v.1.48u (National Institutes of Health, Bethesda, MD, USA) was used to quantify the intensity of protein bands.

Statistical analysis. The data are presented as the mean \pm standard deviation of multiple independent experiments. One-way analysis of variance was used to compare group means, followed by Dunnett's t-test, using StataSE12 software (Stata Corp., College Station, TX, USA). $\mathrm{P}<0.05$ was considered to indicate a statistically significant difference.

\section{Results}

Selection of quinine using computational virtual screening. The 1,792 compounds were docked into the binding pocket of TRAF6 using AutoDock 4.10 software, and the top 50 compounds were selected with the lowest estimated inhibition constant. Subsequently, binding conformations for each of these selected compounds were exported and evaluated manually, according to the estimated binding free energy and hydrophobic interactions. Quinine $(\Delta \mathrm{G}=-6.8 \mathrm{kcal} / \mathrm{mol})$ was selected based on these calculations and purchased for further experimental testing.

Fig. 1 demonstrated the binding mode of quinine: The oxygen atom of the hydroxyl group of quinine forms H-bonds to the OD1 atom of residue Asp-57; the quinoline ring is in $\pi-\pi$ stacking interaction with Phe-60 and also in good hydrophobic interactions with the surrounding residues, Phe-60, Pro-63 and Leu-64.

Quinine suppresses the growth of A549 and HeLa cells. As a result of the molecular docking results, which suggested quinine binds to TRAF6 and affects its function, the present study therefore used HeLa and A549 cell lines that express high levels of endogenous TRAF6 $(5,10)$ in the following experiments. As shown in Fig. 2A, quinine inhibited HeLa cell proliferation in a concentration- and time-dependent manner, with concentrations $>5 \mu \mathrm{M}$ markedly reducing cell viability. An increase in the concentration between 10 and $500 \mu \mathrm{M}$ increased the inhibition rate from 12.80 to $82.53 \%$, when treated for $72 \mathrm{~h}$. Similarly, prolonged incubation with quinine
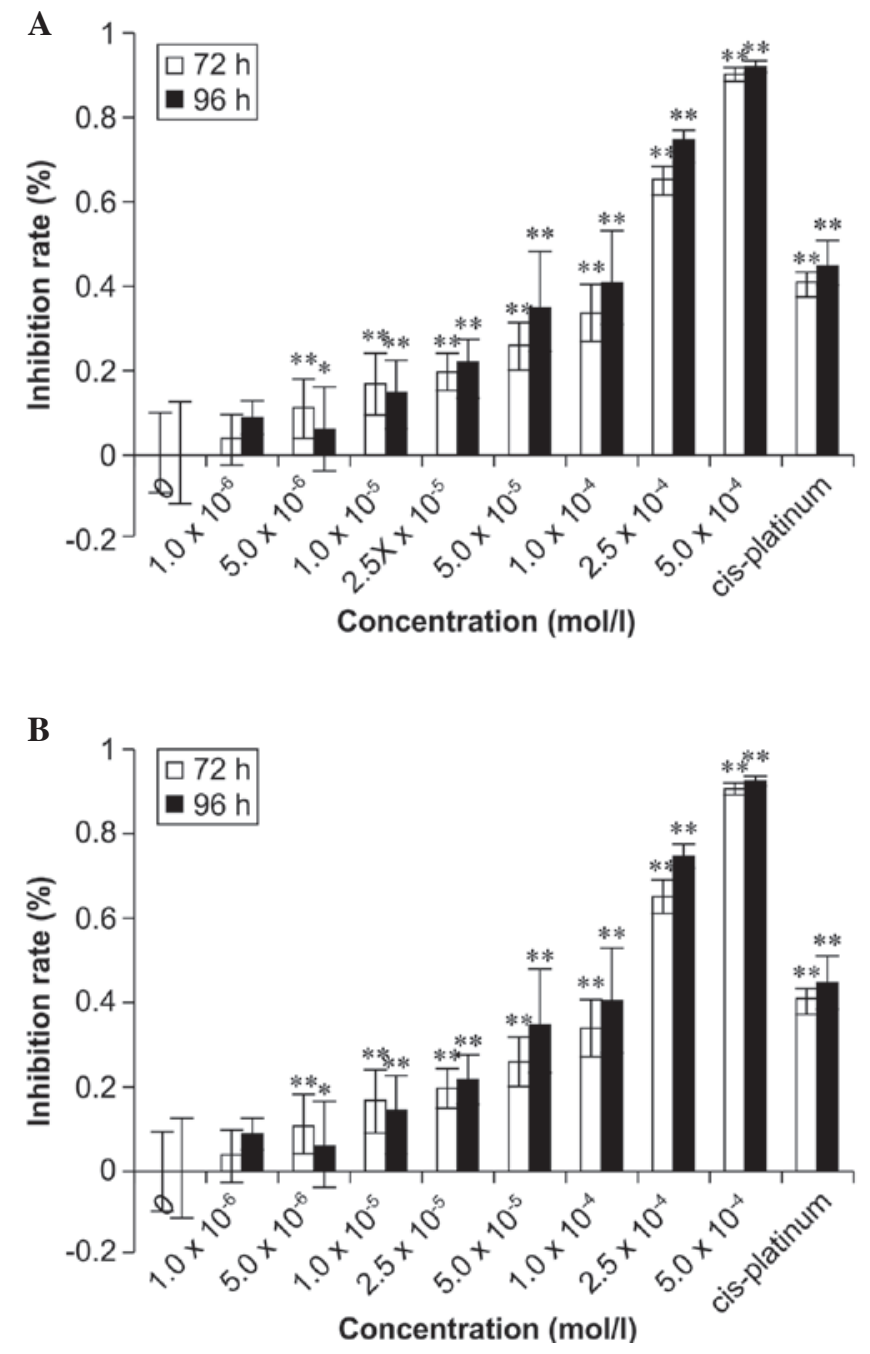

Figure 2. Inhibitory effects of quinine on tumor cell lines. (A) HeLa and (B) A549 cells were seeded into 96-well plates and were treated with various concentrations of quinine for 72 and $96 \mathrm{~h}$. Cell viability was evaluated with using a 3-(4,5-dimethylthiazol-2-yl)-2,5-diphenyltetrazolium bromide assay. The results are presented as the mean \pm standard deviation $(n=3$; ${ }^{*} \mathrm{P}<0.05$ and ${ }^{* * *} \mathrm{P}<0.01$ vs. the control).

for $96 \mathrm{~h}$ increased the rate of inhibition from 17.67 to $89.73 \%$ when the dose was increased between 10 and $500 \mu \mathrm{M}$. These effects were also reproduced in the A549 cells (Fig. 2B).

Quinine induces apoptosis in HeLa cells. The present study next investigated whether the decreased cell viability following quinine treatment was due to the occurrence of apoptosis. To confirm this hypothesis, annexin V-FITC and PI staining were used to discriminate between cells undergoing early apoptosis (annexin V+/PI-) and necrosis (PI+). The percentage of apoptotic cells increased in a dose-dependent manner upon quinine treatment (Fig. 3). The fraction of viable cells decreased from 91.28 down to $42.80 \%$ following treatment with $200 \mu \mathrm{M}$ quinine. This was simultaneously accompanied with an increase (5.64 to 49.83\%) in the fraction of early apoptotic cells in the population. Treatment with the positive control, Cis-platinum, reduced the number of viable cells to $64.99 \%$ and was accompanied by a concomitant increase in the fraction of early apoptotic cells to $29.94 \%$. By contrast, the percentage of PI+ cells increased from 0.92 to $6.48 \%$ upon 
A

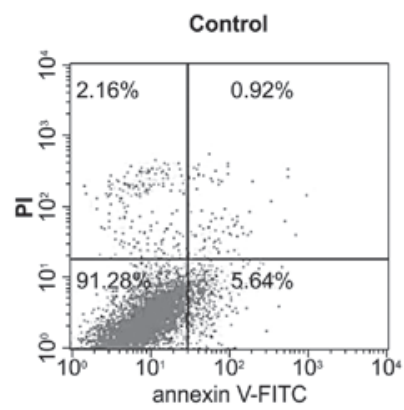

Cis-platinum $1.67 \times 10^{-5} \mathrm{~mol} / \mathrm{l}$

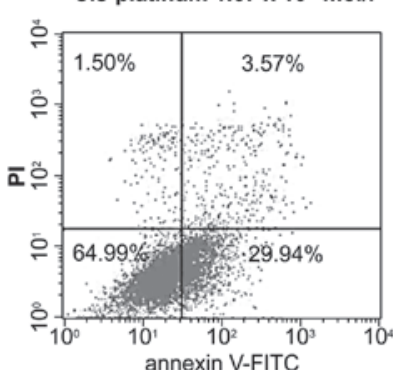

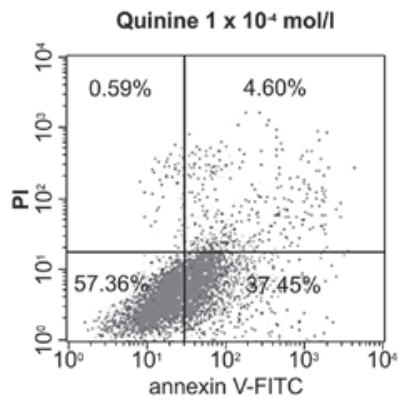
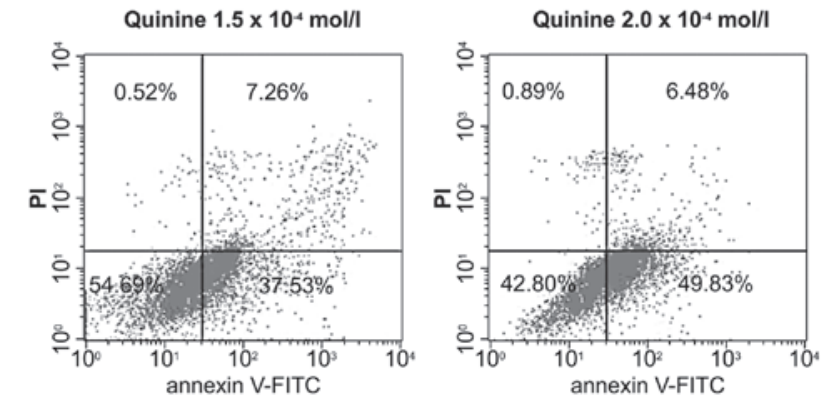

B

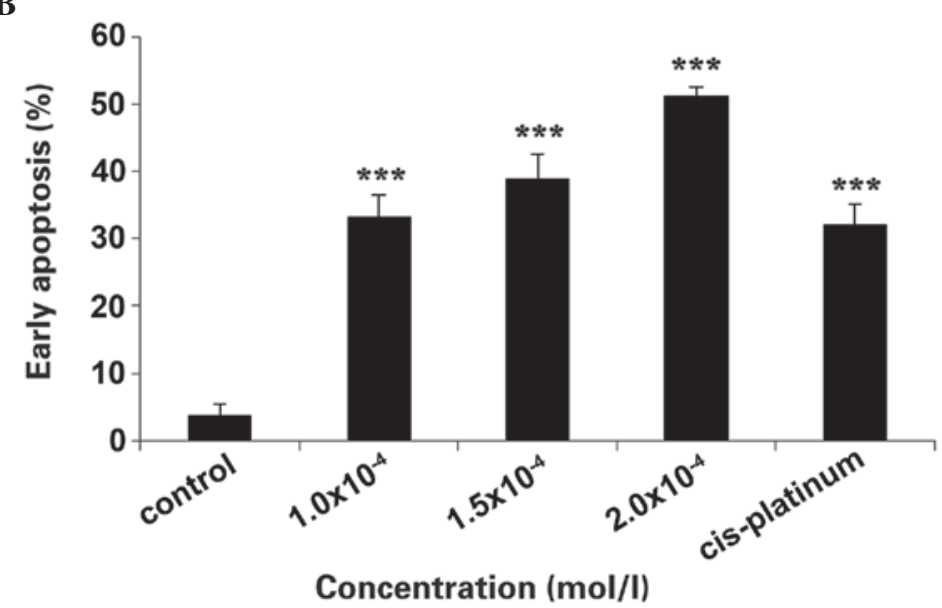

Figure 3. Evaluation of apoptosis by flow cytometry after Annexin V-FITC double-staining. Negative and positive control cells received no drug treatment or were treated with cis-platinum, respectively. HeLa cells were treated with varying concentrations of quinine for 48 h. (A) The upper left quadrant of each scatterplot shows necrotic cells; the upper right quadrant shows late apoptotic or necrotic cells; the lower left quadrant shows viable cells; and the lower right quadrant shows early apoptotic cells. (B) Quantitative analysis of flow cytometry results, represented as the mean \pm standard deviation of three independent experiments. Means were compared by one-way analysis of variance followed by Dunnett's t test $\left(^{* * *} \mathrm{P}<0.001\right.$ vs. control). FITC, fluorescein isothiocyanate; PI, propidium iodide.

treatment with $200 \mu \mathrm{M}$ quinine. These results indicated that quinine induces cell apoptosis, rather than necrosis.

Quinine-induced apoptosis activates BAX in HeLa and A549 cells. The above results showed that quinine application resulted in the inhibition of HeLa and A549 cell growth and proliferation, and induced early apoptosis. Since the BCL-2 family serves a critical role in the execution of programmed cell death, the present study assessed whether the apoptosis induced by quinine was associated with changes in the levels of either BCL-2 or BAX (Fig. 4). Indeed, the quinine treatment led to an increase in the levels of the pro-apoptotic factor, BAX, in a time-dependent manner, as well as a decrease in the expression of BCL-2. These results suggested that quinine exposure is responsible for inducing apoptosis, which underlies the reduced viability of HeLa and A549 cells.

Quinine treatment regulates the phosphorylation of AKT. To elucidate the underlying molecular mechanism in which quinine induces apoptosis, the phosphorylation status of AKT was analyzed in these two cell lines following LPS treatment. The present study decided to incorporate LPS here, since it is a robust inducer of AKT activity in HeLa and A549 cells $(11,12)$. Stimulation of HeLa and A549 cells with LPS for $3 \mathrm{~h}$ resulted in an increase in p-AKT levels, and this effect was inhibited by quinine treatment (Fig. 5). In addition, HeLa and A549 cell proliferation was enhanced by treatment with LPS, which was 

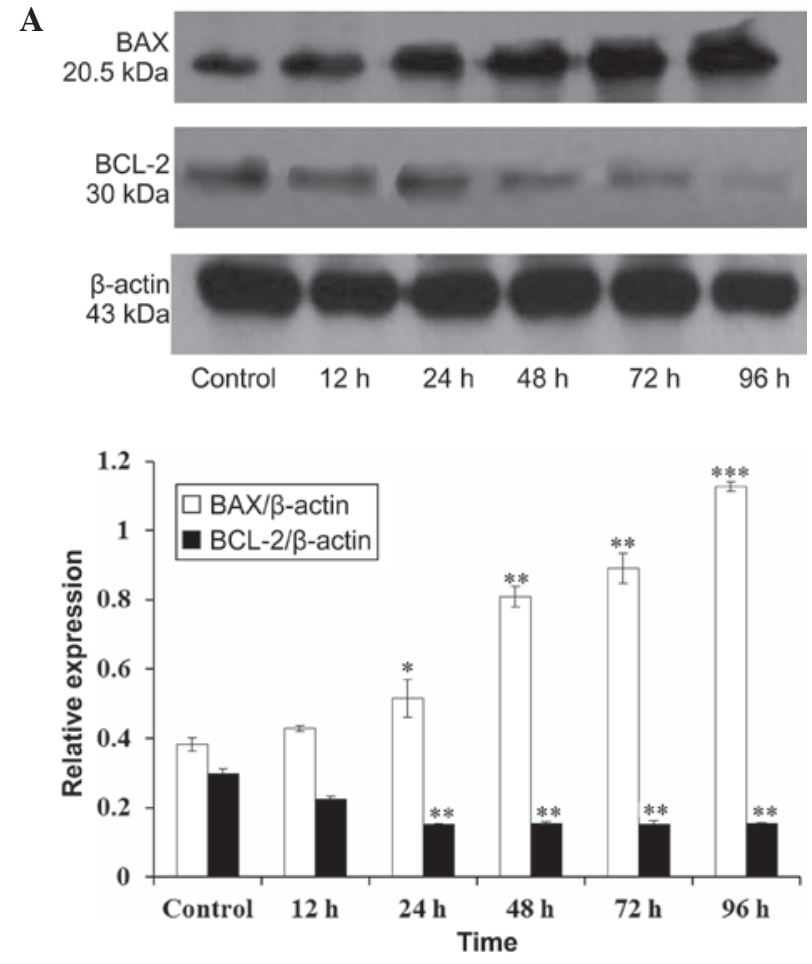

B
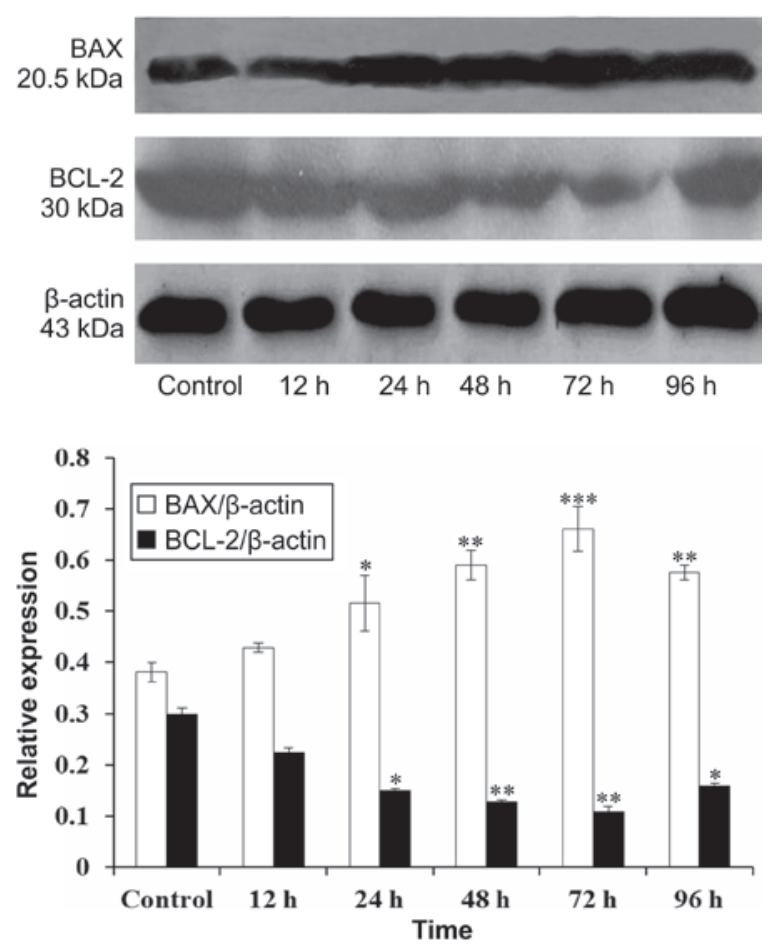

Figure 4. Quinine modulates the expression levels of BCL-2 and BAX in HeLa and A549 cells. (A) HeLa and (B) A549 cells were treated with quinine $\left(1.5 \times 10^{-4} \mathrm{~mol} / \mathrm{l}\right)$ for the indicated durations. Western blotting was subsequently performed to determine the expression of BCL-2 and BAX in the cell lines. Densitometric analysis was performed to quantify the levels of the proteins. The data are expressed as the mean \pm standard deviation $(\mathrm{n}=2$ ${ }^{*} \mathrm{P}<0.05,{ }^{* *} \mathrm{P}<0.01$ and ${ }^{* * *} \mathrm{P}<0.001$ vs. control). BCL, B-cell lymphoma; BAX, BCL-2-associated $\mathrm{X}$ protein.

abrogated in the presence of quinine (Fig. 6). These results indicated that quinine induced apoptosis via the activation of AKT.

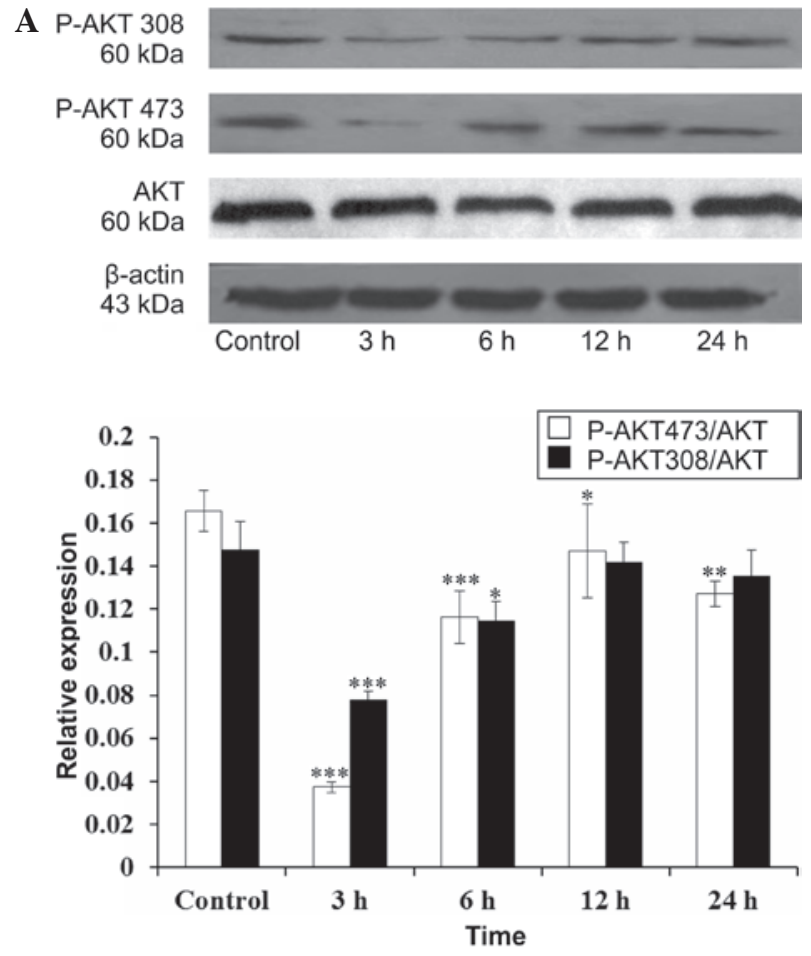

B
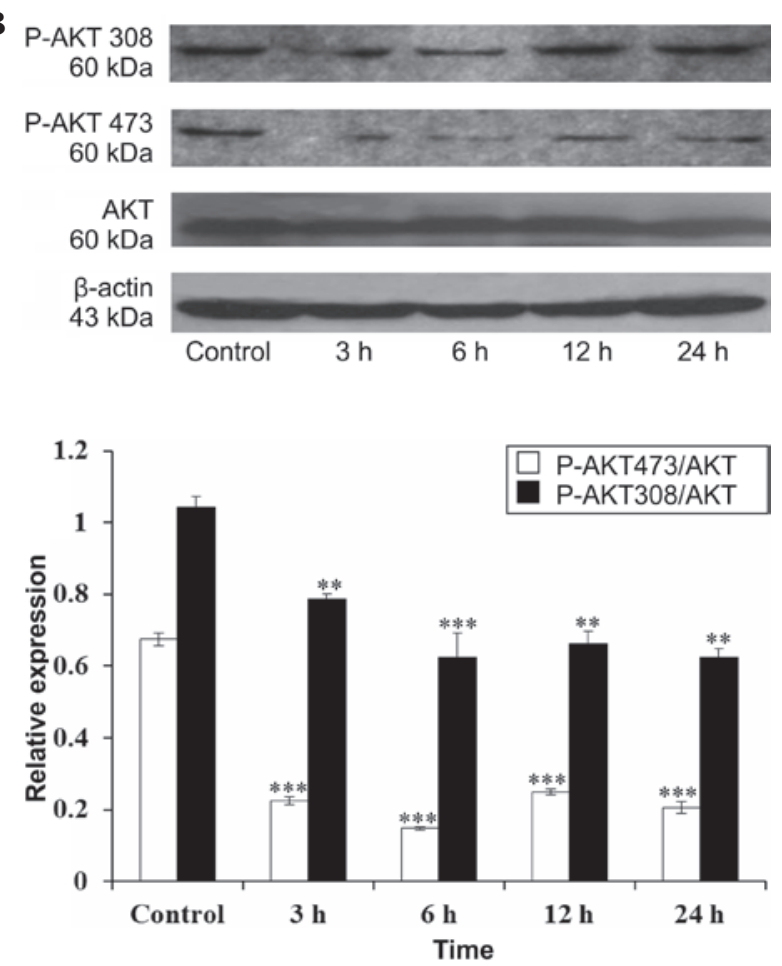

Figure 5. Effect of quinine on LPS-mediated AKT activation. (A) HeLa and (B) A549 cells were pre-treated with quinine for the indicated durations, and were subsequently treated with LPS $(20 \mu \mathrm{g} / \mathrm{ml})$ for $3 \mathrm{~h}$. Western blotting was performed to determine the protein expression levels of p-AKT (Thr308 and Ser473). Total AKT was used as a loading control. The data are expressed as the mean \pm standard deviation $\left(\mathrm{n}=2 ;{ }^{*} \mathrm{P}<0.05,{ }^{* *} \mathrm{P}<0.01\right.$ and ${ }^{* * * *} \mathrm{P}<0.001$ vs. control). LPS, lipopolysaccharide; AKT, protein kinase B; p-, phosphorylated.

\section{Discussion}

Cancer has been the top health threat for over a century and is second only to cardiovascular diseases in mortality. AKT 

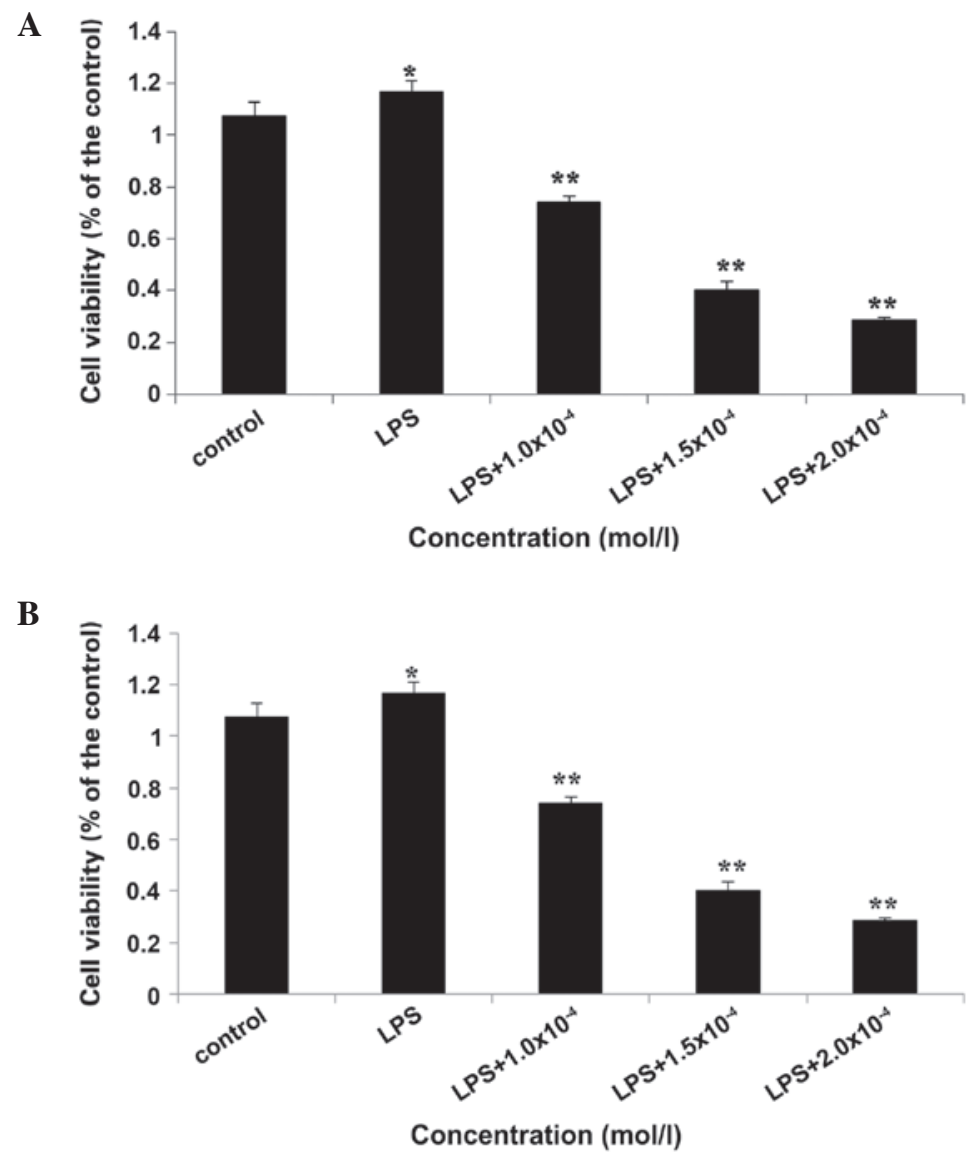

Figure 6. Effect of LPS on quinine-induced cytotoxicity in tumor cell lines. (A) HeLa and (B) A549 cells were seeded into 96-well plates and treated with LPS $(20 \mu \mathrm{g} / \mathrm{ml})$ in the absence or presence of quinine at concentrations of 100,150 or $200 \mu \mathrm{M}$ for $72 \mathrm{~h}$. Cell viability was evaluated using a 3-(4,5-dimethylthiazol-2-yl)-2,5-diphenyltetrazolium bromide assay. The data are presented as the mean \pm standard deviation $\left(\mathrm{n}=3\right.$; ${ }^{*} \mathrm{P}<0.05$ and ${ }^{* *} \mathrm{P}<0.01 \mathrm{vs}$. control). LPS, lipopolysaccharide.

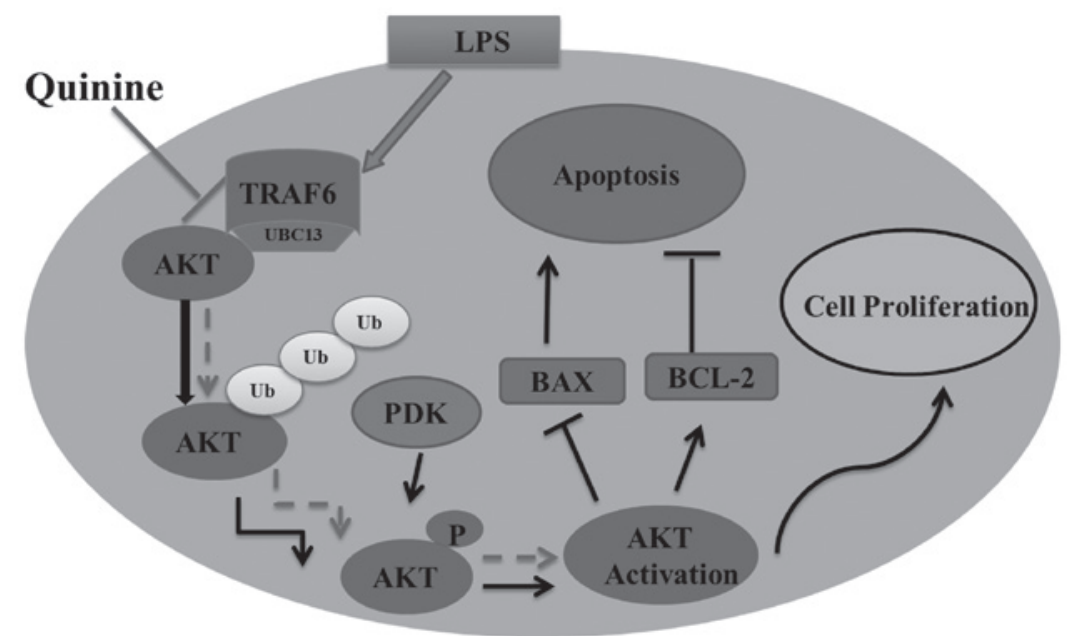

Figure 7. Model for the inhibition of AKT signaling by quinine. Stimuli, including the bacterial endotoxin LPS leads to the activation of the E3 ubiquitin ligase, TRAF6, via a series of cytokines. Through conjugation of K63-linked ubiquitin chains, TRAF6 enhances AKT activity. Two major consequences of AKT signaling are the suppression of apoptosis, via the modulation of BCL-2 family members, and enhanced proliferation. Positive regulation of AKT by TRAF6 was inhibited by the antimalarial compound, quinine. The consequent suppression of BCL-2 and upregulation of BAX sensitizes cells to apoptosis. LPS, lipopolysaccharide; TRAF6, tumor necrosis factor receptor-associated factor 6; AKT, protein kinase B; BCL, B-cell lymphoma; BAX, Bcl-2-associated X protein; p, phosphate; Ub, ubiquitin; UBC, Ub C.

is activated in $>50 \%$ of human cancer cell lines. The AKT signaling pathway has been linked to a variety of cellular processes, including proliferation and migration, and it also serves a role in response to anticancer therapeutics $(13,14)$. An important step in AKT activation is its phosphorylation, and this activation is subsequently enhanced by TRAF6-dependent 
ubiquitination. Yang et al (4) demonstrated that the formation of a stable complex between AKT and TRAF6 is responsible for the enhanced activation. Previous evidence suggested that TRAF6 is associated with tumors, since TRAF6 depletion in osteosarcoma cells was found to reduce $\mathrm{NF}-\kappa \mathrm{B}$ expression and the survival rate for cancer patients overexpressing TRAF6 is relatively low (5,10-17). Therefore, targeting TRAF6-mediated AKT activation may constitute an excellent therapeutic strategy for the treatment of cancer.

The present study used computational methods to select small molecules, which can bind with TRAF6, and pursued ex vivo assays to evaluate their antitumor activities and to investigate possible mechanisms of action. Firstly, the computational docking data revealed that quinine may be an excellent ligand for TRAF6, thereby representing a potential candidate for modulating TRAF6-mediated AKT activation. Subsequently, the antitumor activity of quinine was assessed and it was revealed that this compound inhibited the growth of HeLa and A549 cells in a dose-dependent manner. Secondly, it is well-known that apoptosis is closely associated with the initiation, progression and metastasis of cancer, as well as the elimination of malignant or infected cells (18-21). Apoptosis provides a vital homeostatic mechanism for maintaining an appropriate number of cells in the body through a balance of cell division and cell death (22). Consequently, the present study evaluated the cytotoxic and apoptotic effects of quinine through appropriately selected cell lines and found that quinine caused a significant loss of cell viability in HeLa and A549 cells in a concentration- and time-dependent manner. Under these experimental conditions, the loss of cell viability following quinine treatment was predominantly due to the induction of apoptosis.

Next, to further investigate the underlying mechanism of quinine-induced apoptosis, western blotting was used to analyze the expression levels of BCL-2 and BAX in HeLa and A549 cells. Quinine was demonstrated to suppress the expression of BCL-2, whilst stimulating that of BAX in a time-dependent manner. These results supported that quinine induces apoptosis in HeLa and A549 cells via modulation of these key apoptosis-regulating proteins. It has been previously suggested that mitochondria serve a key role in cell apoptosis, since numerous BCL-2 family proteins interact with the mitochondrial outer membrane $(23,24)$. The mutual antagonism that exists between subsets of BCL-2 family members, including BCL-2 and BAX, directly impacts the mitochondrial membrane permeability, which ultimately determines whether cytochrome $c$ is released in order to activate caspases and induce apoptosis (25).

AKT can also directly or indirectly affect mitochondria-dependent apoptosis via the modulation of BCL-2 family proteins. Previous evidence demonstrates that phosphorylation of AKT can inactivate BAX $(26,27)$. Others have found that activated AKT can phosphorylate BCL-2-associated death domain (BAD) at Ser136, and the association of p-BAD with 14-3-3 proteins can inhibit apoptosis by preventing p-BAD from interacting with the antiapoptotic BCL-2 family members, including BCL-2 or BCL-extra large (28). These collective observations along with the present results indicated that activated AKT can attenuate mitochondria-dependent apoptosis by inactivating BAD, BAX and other forkhead tran- scription factors, and that quinine can counter this process by inhibiting the activation of AKT.

Additionally, while serving a critical role in the regulation of cell survival and apoptosis in a variety of cell types (29-31), AKT activation is accomplished via the phosphorylation at Ser473 in the C-terminal hydrophobic region by phosphoinositide-dependent kinase (PDK)-2 and at Thr308 in the catalytic domain by PDK-1 (32). Through computational virtual docking, the present study quickly identified appropriate ligands, including quinine, which can bind effectively with TRAF6, and subsequently, detected the effect of quinine on the activity of AKT. These experimental results demonstrated that quinine can inhibit the phosphorylation of AKT at both Thr-308 and Ser-473, thereby suggesting that quinine-induced apoptosis is likely a result of inhibition of AKT phosphorylation.

In conclusion, the present study demonstrated that quinine can reduce cell viability and induce apoptosis in HeLa and A549 cells. These novel activities were associated with the inhibition of AKT activation by inhibiting its phosphorylation at Thr-308 and Ser-473, as well as AKT-induced suppression of BCL-2 and upregulation of BAX (Fig. 7). Further investigations are currently underway to determine whether quinine is a viable anticancer agent and whether TRAF6 can serve as a therapeutic target for developing novel anticancer drugs.

\section{Acknowledgements}

The preset study was supported by the Tianjin Research Program of Applied Basic Science and Advanced Technology (no. 14JCZDJC33600). The authors would like to thank The Scripps Research Institute for making available the AutoDock docking program and supporting literature, and the PDB database for free access to the X-ray crystal structure of TRAF6.

\section{References}

1. Bae S, Kim SY, Jung JH, Yoon Y, Cha HJ, Lee H, Kim K, Kim J, An IS, Kim J, et al: Akt is negatively regulated by the MULAN E3 ligase. Cell Res 22: 873-885, 2012.

2. Cai J, Yang J and Jones DP: Mitochondrial control of apoptosis: The role of cytochrome $c$. Biochim Biophys Acta 1366: 139-149, 1998.

3. Jeong SJ, Pise-Masison CA, Radonovich MF, Park HU and Brady JN: Activated AKT regulates NF-kappaB activation, p53 inhibition and cell survival in HTLV-1-transformed cells. Oncogene 24: 6719-6728, 2005.

4. Yang WL, Wang J, Chan CH, Lee SW, Campos AD, Lamothe B, Hur L, Grabiner BC, Lin X, Darnay BG and Lin HK: The E3 ligase TRAF6 regulates Akt ubiquitination and activation. Science 325: 1134-1138, 2009.

5. Zhong L, Cao F and You Q: Effect of TRAF6 on the biological behavior of human lung adenocarcinoma cell. Tumor Biol 34: 231-239, 2013.

6. Deng L, Wang C, Spencer E, Yang L, Braun A, You J, Slaughter C, Pickart $\mathrm{C}$ and Chen $\mathrm{ZJ}$ : Activation of the IkappaB kinase complex by TRAF6 requires a dimeric ubiquitin-conjugating enzyme complex and a unique polyubiquitin chain. Cell 103: 351-361, 2000.

7. Feng H, Lopez GY, Kim CK, Alvarez A, Duncan CG, Nishikawa R, Nagane M, Su AJ, Auron PE, Hedberg ML, et al: EGFR phosphorylation of DCBLD2 recruits TRAF6 and stimulates AKT-promoted tumorigenesis. J Clin Invest 124: 3741-3756, 2014.

8. Wang DH, Qu WL, Shi LQ and Wei J: Molecular docking and pharmacophore model studies of Rho kinase inhibitors. Mol Simulat 37: 488-494, 2011. 
9. Pettersen EF, Goddard TD, Huang CC, Couch GS, Greenblatt DM, Meng EC and Ferrin TE: UCSF chimera-A visualization system for exploratory research and analysis. J Comput Chem 25: 1605-1612, 2004.

10. Hartupee J, Liu C, Novotny M, Sun D, Li X and Hamilton TA IL-17 signaling for mRNA stabilization does not require TNF receptor-associated factor 6. J Immunol 182: 1660-1666, 2009.

11. Elumalai P, Arunkumar R, Benson CS, Sharmila G and Arunakaran J: Nimbolide inhibits IGF-I-mediated PI3K/Akt and MAPK signalling in human breast cancer cell lines (MCF-7 and MDA-MB-231). Cell Biochem Funct 32: 476-484, 2014.

12. Schnetzke U, Fischer M, Kuhn AK, Spies-Weisshart B, Zirm E, Hochhaus A, Müller JP and Scholl S: The E3 ubiquitin ligase TRAF6 inhibits LPS-induced AKT activation in FLT3-ITD-positive MV4-11 AML cells. J Cancer Res Clin Oncol 139: 605-615, 2013.

13. Gills JJ and Dennis PA: The development of phosphatidylinositol ether lipid analogues as inhibitors of the serine/threonine kinase, Akt. Expert Opin Investig Drugs 13: 787-797, 2004.

14. Luo J, Manning BD and Cantley LC: Targeting the PI3K-Akt pathway in human cancer: Rationale and promise. Cancer Cell 4: 257-262, 2003.

15. Nishimura $\mathrm{M}$ and Naito $\mathrm{S}$ : Tissue-specific mRNA expression profiles of human toll-like receptors and related genes. Biol Pharm Bull 28: 886-892, 2005.

16. Mao R, Fan Y, Mou Y, Zhang H, Fu S and Yang J: TAK1 lysine 158 is required for TGF- $\beta$-induced TRAF6-mediated Smad-independent IKK/NF- $\kappa \mathrm{B}$ and JNK/AP-1 activation. Cell Signal 23: 222-227, 2011

17. Chantzoura E, Prinarakis E, Panagopoulos D, Mosialos G and Spyrou G: Glutaredoxin-1 regulates TRAF6 activation and the IL-1 receptor/TLR4 signalling. Biochem Biophys Res Commun 403: 335-339, 2010

18. Golbano JM, Lóppez-Aparicio P, Recio MN and Pérez-Albarsanz MA: Finasteride induces apoptosis via Bcl-2, $\mathrm{Bcl}-\mathrm{xL}, \mathrm{Bax}$ and caspase-3 proteins in LNCaP human prostate cancer cell line. Int J Oncol 32: 919-924, 2008.

19. Alonso-Castro AJ, Ortiz-Sánchez E, García-Regalado A, Ruiz G, Núñez-Martínez JM, González-Sánchez I, Quintanar-Jurado V, Morales-Sánchez E, Dominguez F, López-Toledo G, et al: Kaempferitrin induces apoptosis via intrinsic pathway in HeLa cells and exerts antitumor effects. J Ethnopharmacol 145: 476-489, 2013
20. Xu C, Wu A, Zhu H, Fang H, Xu L, Ye J and Shen J: Melatonin is involved in the apoptosis and necrosis of pancreatic cancer cell line SW-1990 via modulating of Bcl-2/Bax balance. Biomed Pharmacother 67: 133-139, 2013.

21. Costa LS, Telles CB, Oliveira RM, Nobre LT, Dantas-Santos N, Camara RB, Costa MS, Almeida-Lima J, Melo-Silveira RF, Albuquerque IR, et al: Heterofucan from Sargassum filipendula induces apoptosis in HeLa Cells. Mar Drugs 9: 603-614, 2011.

22. $\mathrm{Hu} \mathrm{W}$ and Kavanagh JJ: Anticancer therapy targeting the apoptotic pathway. Lancet Oncol 4: 721-729, 2003.

23. Yang J, Liu X, Bhalla K, Kim CN, Ibrado AM, Cai J, Peng TI, Jones DP and Wang X: Prevention of apoptosis by Bcl-2: Release of cytochrome $c$ from mitochondria blocked. Science 275: 1129-1132, 1997.

24. Kluck RM, Bossy-Wetzel E, Green DR and Newmeyer DD: The release of cytochrome $\mathrm{c}$ from mitochondria: A primary site for Bcl-2 regulation of apoptosis. Science 275: 1132-1136, 1997.

25. Zhang L, Xing D and Chen MJ: Bim(L) displacing Bcl-x(L) promotes bax translocation during TNF alpha-induced apoptosis. Apoptosis 13: 950-958, 2008.

26. Gardai SJ, Hildeman DA, Frankel SK, Whitlock BB, Frasch SC, Borregaard N, Marrack P, Bratton DL and Henson PM: Phosphorylation of Bax Ser184 by Akt regulates its activity and apoptosis in neutrophils. J Biol Chem 279: 21085-21095, 2004.

27. Linseman DA, Butts BD, Precht TA, Phelps RA, Le SS, Laessig TA, Bouchard RJ, Florez-McClure ML and Heidenreich KA: Glycogen synthase kinase-3 beta phosphorylates bax and promotes its mitochondrial localization during neuronal apoptosis. J Neurosci 24: 9993-10002, 2004.

28. Zha J, Harada H, Yang E, Jockel J and Korsmeyer SJ: Serine phosphorylation of death agonist BAD in response to survival factor results in binding to 14-3-3 not BCL-XL. Cell 87: 619-628, 1996.

29. Marte BM and Downward J: PKB/Akt: Connecting phosphoinositide 3-kinase to cell survival and beyond. Trends Biochem Sci 22: 355-358, 1997.

30. Datta SR, Brunet A and Greenberg ME: Cellular survival: A play in three Akts. Genes Dev 13: 2905-2927, 1999.

31. Song G, Ouyang G and Bao S: The activation of Akt/PKB signaling pathway and cell survival. J Cell Mol Med 9: 59-71, 2005.

32. Scheid MP and Woodgett JR: Unravelling the activation mechanisms of protein kinase B/Akt. FEBS Lett 546: 108-112, 2003. 